\title{
An unusual case of bilateral renal lymphangiectasia
}

\author{
Authors: \\ Shailender N. Singh ${ }^{1}$ \\ Gaurav Sundar ${ }^{1}$ \\ Hanuman Satishchandra ${ }^{1}$ \\ Affiliations: \\ ${ }^{1}$ Bangalore Medical College \\ and Research Institute, \\ Karnataka, India \\ Correspondence to: \\ Gaurav Sundar \\ Email: \\ gauravsundar@gmail.com \\ Postal address: \\ B105 Sriramsrishti \\ Apartments, Anandnagar, \\ Bangalore 560032, India \\ Dates: \\ Received: 05 Mar. 2014 \\ Accepted: 05 Mar. 2014 \\ Published: 08 Dec. 2014 \\ How to cite this article: \\ Singh SN, Sundar G, \\ Satishchandra H. An unusual \\ case of bilateral renal \\ lymphangiectasia. S Afr J \\ Rad. 2014;18(1); Art. \#587, \\ 4 pages. http://dx.doi/ \\ org/10.4102/sajr.v18i1.587

\section{Copyright:} \\ (C) 2014. The Authors. \\ Licensee: AOSIS \\ OpenJournals. This work is \\ licensed under the Creative \\ Commons Attribution \\ License.
}

Read online:
Renal lymphangiectasia is a very rare benign disorder characterised by dilatation of the perirenal and peripelvic lymphatics. The condition is commonly misdiagnosed for other cystic lesions of the kidney such as polycystic kidneys. The diagnosis can be made with near-certainty if classical imaging findings are recognised. We report a case of bilateral renal lymphangiectasia, diagnosed on ultrasonography and computed tomography based on the typical imaging findings and the laboratory analysis of aspirated fluid. The patient was managed conservatively.

\section{Introduction}

Renal lymphangiectasia is a rare benign disorder of the kidneys with dilated perirenal lymphatics caused by non-communication of the perirenal and peripelvic lymphatics with the main lymphatics. ${ }^{1}$ The condition in itself is benign, but is commonly misdiagnosed as other cystic renal diseases and hydronephrosis. ${ }^{2}$ Knowledge of this condition and its typical imaging appearances will assist radiologists to arrive at the correct diagnosis and permit appropriate management of the patient. We present a case of bilateral renal lymphangiectasia associated with a right-sided retrocaval ureter with typical ultrasonography (US) and computed tomography (CT) findings. To the best of our knowledge, this is the first reported association of bilateral renal lymphangiectasia with a retrocaval ureter.

\section{Ethical considerations}

Ethical clearance was obtained from the institutional review board.

\section{Case presentation}

A 24-year-old male presented with complaints of abdominal pain and distension, which he had experienced for two years. He had an episode of haematuria one week prior to presentation. He had no history of fever, diabetes mellitus or any previous abdominal surgeries. On clinical examination he was hypertensive with a blood pressure of 150/110 $\mathrm{mmHg}$ and had abdominal ascites.

Routine laboratory investigations revealed his haemoglobin, total leucocyte count and differential leucocyte counts to be within normal limits. His blood urea nitrogen level was $7.14 \mathrm{mmol} / \mathrm{L}$ (normal range: 8.0-16.4 mmol/L), serum creatinine $79.56 \mu \mathrm{mol} / \mathrm{L}$ (normal range: 50-110 $\mu \mathrm{mol} / \mathrm{L}$ ) and blood urea nitrogen to creatinine ratio 22.2 (normal value: $<10: 1$ ), indicating prerenal azotaemia. His urine examination was normal. The patient subsequently underwent imaging to assess the abdominal pain and distention.

Ultrasonography examination of the abdomen using a 2-5 MHz curvilinear transducer (Philips iU-22, Eindhoven, Netherlands) demonstrated bilaterally enlarged and echogenic kidneys with loss of corticomedullary differentiation. There were large, clear, multi-septated fluid collections in the perinephric spaces bilaterally, which were indenting the surface of the kidney, and mild hydronephrosis on the right side (Figure 1). There was mild to moderate ascites. No cysts or masses were identified in other organs.

A contrast enhanced CT of the abdomen was performed on a six-slice helical scanner (Siemens Somatom Emotion 6, Munich, Germany) to investigate the extent and relation of the fluid collection to adjacent structures. This revealed enlarged kidneys with preserved reniform shape as well as septated perinephric collections (Figure 2). The two fluid collections were communicating with each other across the midline, anterior to the inferior vena cava and aorta (Figure 3 ). The density of the fluid collection ranged from 3-10 Hounsfield units. No soft tissue or fat density masses 
were present within the fluid collections. Both ureters were delineated throughout their course and a retrocaval ureter was noted on the right side coursing medially, posterior to the inferior vena cava at the level of the renal hilum and coursing back laterally (distally). On the right side, there was hydronephrosis and blunting of the calyces secondary to the retrocaval ureter (Figure 2).

There was no hydronephrosis on the left side. Renal arteries and veins were normal on both sides.
Both kidneys showed normal contrast enhancement and excretion.

The perirenal fluid, aspirated under ultrasound guidance, was found to be clear and straw coloured. Laboratory analysis of the aspirated fluid revealed urea level of $5.24 \mathrm{mmol} / \mathrm{L}$, total protein of $0.77 \mathrm{~g} / \mathrm{dL}$, absence of triglycerides and the presence of a few lymphocytes. On protein electrophoresis there was a selective protein band in the albumin region. Both gram and acid-fast stain revealed no organisms and

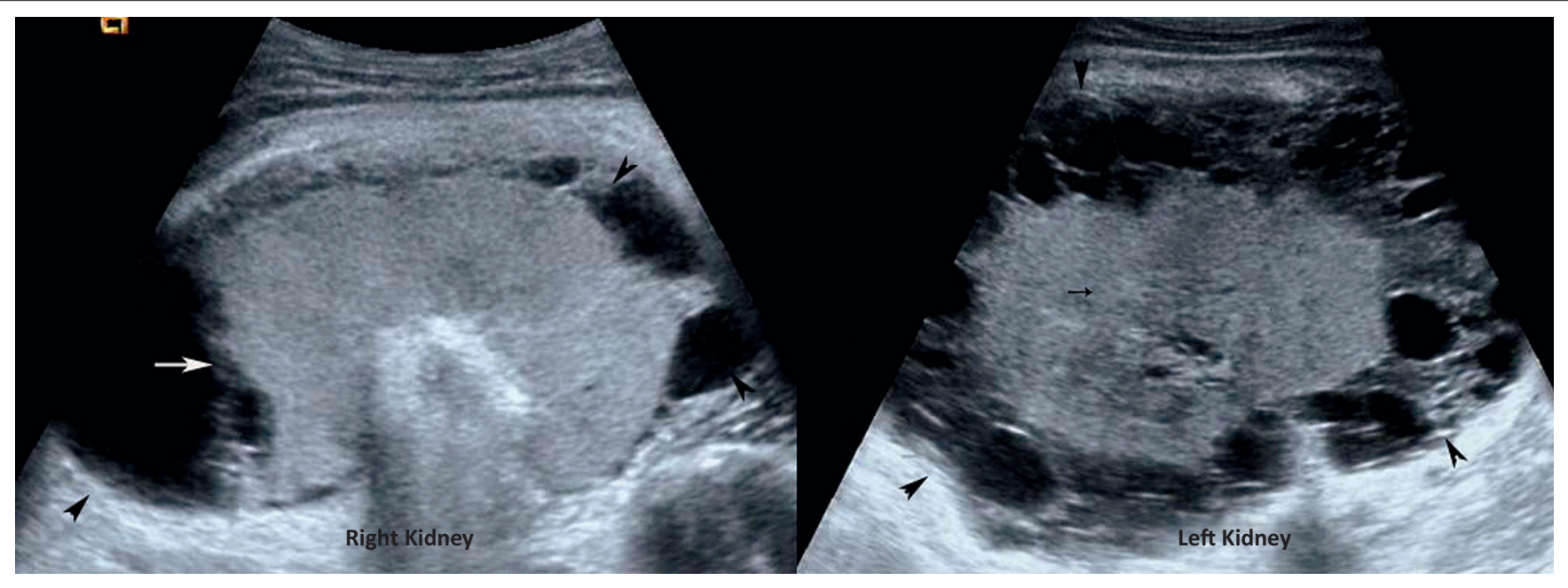

FIGURE 1: Sagittal US image of the kidneys. Bilateral multiseptated perinephric collection is noted (arrowheads). Corticomedullary differentiation is lost (solid black arrow).

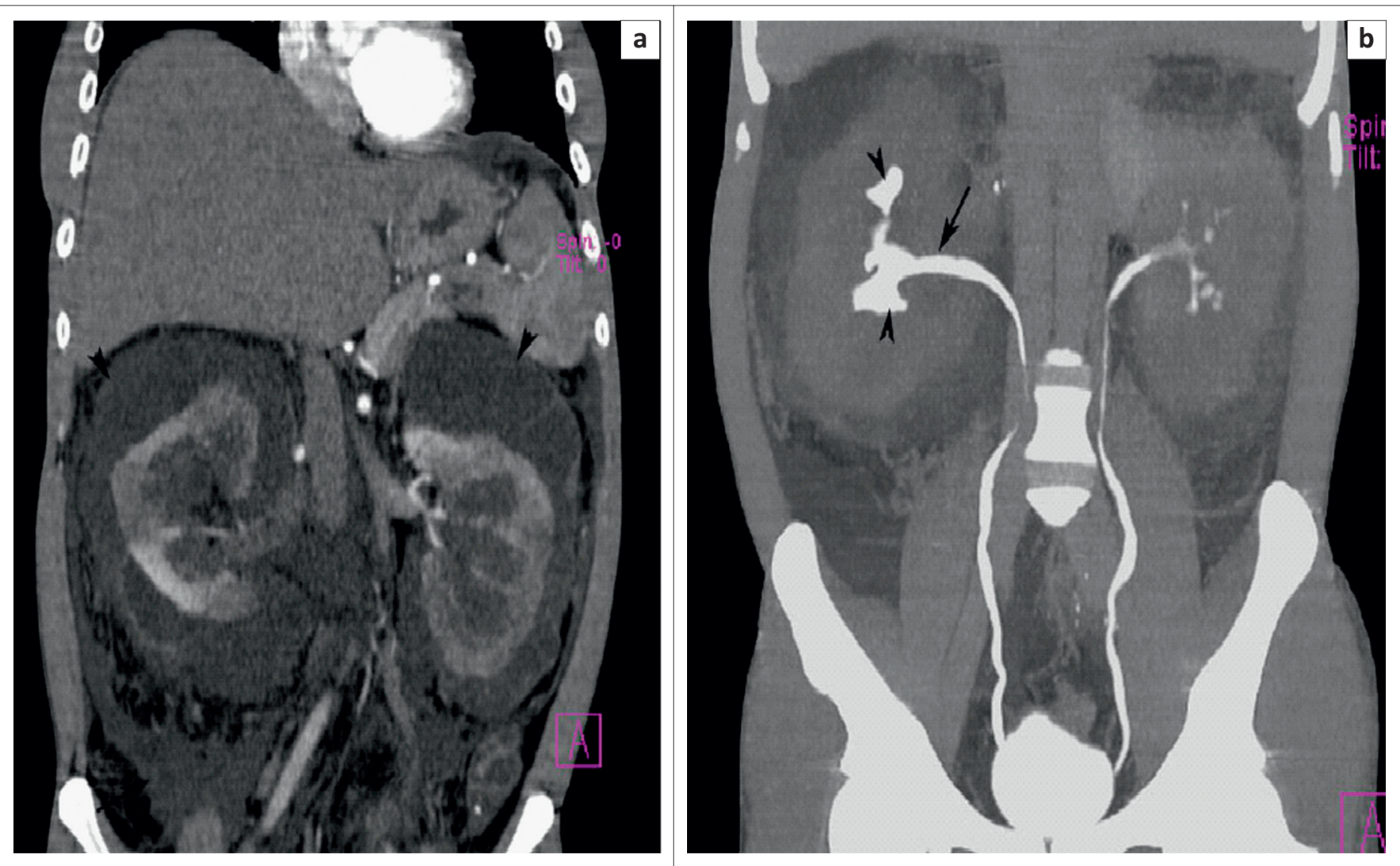

FIGURE 2: Reformatted (a) coronal contrast enhanced CT scan in the corticomedullary phase. Bilateral enlarged kidneys with perinephric collection (arrowheads) Reformatted (b) maximum intensity projection CT urography. Medial deviation of the proximal right ureter posterior to the IVC, swinging back to the normal course distally, consistent with retrocaval ureter (arrow). 


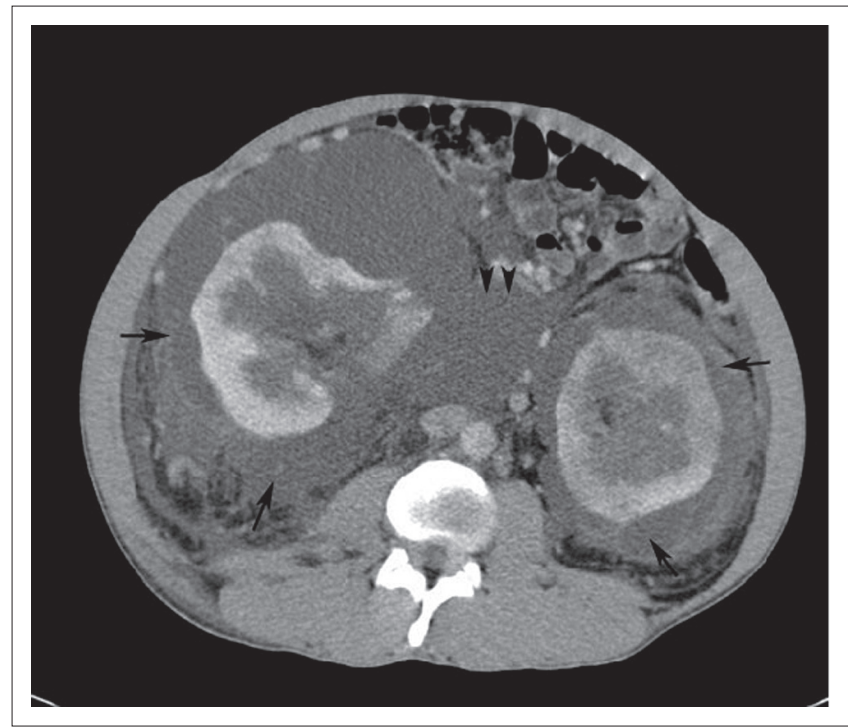

FIGURE 3: Axial contrast-enhanced CT scan. Bilateral low density perinephric collections (black arrows) with normal enhancement of the renal parenchyma and communication of collections with each other across the midline (arrowheads).

culture was negative. The laboratory analysis was in favour of lymphatic fluid.

The diagnosis, based on the typical imaging findings and the laboratory analysis of the fluid, was bilateral renal lymphangiectasia with a retrocaval right ureter. Following conservative management with antihypertensives and diuretics, his renal function improved and blood pressure returned to normal.

\section{Discussion}

Bilateral renal lymphangiectasia is a rare benign condition that is often mistaken for other forms of cystic disease of the kidney. ${ }^{2}$ It is synonymous with renal lymphangiomatosis, hygroma renale and polycystic disease of the renal sinus. ${ }^{1,3,4}$ The exact pathogenesis of this condition is not known but is thought to be due to failure of the perinephric lymphatics to communicate with the main lymphatic system. ${ }^{5}$ There is no particular age or sex predilection for this condition but familial association has been reported in a few cases.

The clinical course of this condition is variable. Most commonly, is asymptomatic and is diagnosed incidentally. However, there can be sudden appearance and rapid growth or cessation of growth and even spontaneous regression of symptoms. ${ }^{5}$ When symptomatic, abdominal pain, haematuria, hypertension and renal failure are usually the presenting features. ${ }^{4}$ Exacerbation during pregnancy has also been reported. ${ }^{1}$ In this case report the patient presented with abdominal pain and distention with hypertension and features of prerenal azotaemia.

Imaging appearances, when typical, can be striking, and often help make the diagnosis. On plain radiography, there is usually a large homogenous soft-tissue mass in the renal fossa displacing the adjacent structures.
Excretory urography may demonstrate hydronephrosis and distortion of the pelvicalyceal system, which may be due to compression of the pelvi-ureteric junction by the collections or associated conditions such as retrocaval ureter, as in our case. ${ }^{6}$ US examination demonstrates multiseptated, thin-walled fluid collections, localised to the perinephric and peripelvic space with normal renal parenchyma. ${ }^{7}$ The kidneys may be of normal size or enlarged and may have increased cortical echogenicity with loss of corticomedullary differentiation due to increased through transmission of US by the collections, as was seen in our case. ${ }^{6}$ It may also appear as a solid mass when the smaller intrarenal lymphatics are blocked. ${ }^{8}$ Ascites may also be present. CT examination may show the presence of low-density fluid collections ( 0 to $10 \mathrm{HU})$ in the perinephric space but the septations may not be delineated as clearly as in US. ${ }^{3}$ There will be no invasion of the adjacent structures or evidence of cysts in other organs. ${ }^{9}$

The differential diagnoses in this condition are polycystic kidney disease, multilocular cystic nephroma, urinoma, abscess and bilateral perirenal liposarcomas. ${ }^{6,7}$ The cysts in polycystic kidney disease and multilocular cystic nephroma are parenchymal. Perirenal liposarcomas show the presence of macroscopic fat. ${ }^{7}$ Apart from the typical clinical history, laboratory analysis of the fluid is helpful to differentiate lymphangiectasia from urinoma and abscess as the former will demonstrate low proteins and fat levels, and a sparse number of lymphocytes. ${ }^{3}$

\section{Management and outcome}

Haematuria, ascites, hypertension and deterioration of the renal function are the known complications of this condition. However, hypertension and renal failure, which is usually mild, will improve following conservative treatment, as in our case. ${ }^{3,9}$ Most asymptomatic patients do not require treatment. In symptomatic patients, especially with large collections, the treatment options available are marsupialisation or nephrostomy. ${ }^{3}$ Antihypertensives and diuretics can be used to reduce blood pressure and fluid secretion. ${ }^{6}$ Nephrectomy is reserved for complicated cases, including Page kidney. ${ }^{6}$

\section{Conclusion}

Our case is of interest as renal lymphangiectasia is a rare benign condition which imaging can differentiate from other cystic diseases of the kidneys and perinephric space. To the best of our knowledge, an association of this condition with retrocaval ureter has not yet been mentioned in the literature. Knowledge of this condition will be helpful in the work-up of cystic diseases of the kidney and in arriving at the correct diagnosis. The diagnosis can be made with near-certainty if typical imaging findings as described here are recognised on US and CT. In difficult cases, an imaging-guided fluid aspiration and laboratory analysis can help to establish the diagnosis and avoid 
unnecessary surgical procedures and their associated risks. Most patients can be managed conservatively and their cases have an uneventful course once the diagnosis is correctly established.

\section{Acknowledgements}

The authors would like to thank Mrs Ranjana for her assistance in English language and grammar in writing the manuscript.

\section{Competing interests}

The authors declare that they have no financial or personal relationship(s) that may have inappropriately influenced them in writing this article.

\section{Authors' contributions}

G.S. (Bangalore Medical College and Research Institute, India) performed the imaging. S.N.S. (Bangalore Medical College and Research Institute, India) and H.S. (Bangalore
Medical College and Research Institute, India) interpreted the imaging studies and wrote the manuscript.

\section{References}

1. Meredith WT, Levine E, Ahlstrom NG, Grantham JJ. Exacerbation of familial renal lymphangiomatosis during pregnancy. AJR Am J Roentgenol. 1988;151:965-6. http://dx.doi.org/10.2214/ajr.151.5.965

2. Lindsey JR. Lymphangiectasia simulating polycystic disease. J Urol 1970; 104:658-662.

3. Schwarz A, Lenz $T$, Klaen R, et al. Hygroma renale: Pararenal lymphatic cysts associated with rennin-dependent hypertension (Page kidney). Case report on bilateral cysts and successful therapy by marsupialization. J Urol 1993;150:953-957.

4. Davidson AJ, Hartman DS. Lymphangioma of the retroperitoneum: CT and sonographic characteristics. Rad 1990;175:507-510. http://dx.doi.org/10.1148/ radiology.175.2.2183287

5. Leonidas JC, Brill PW, Bhan I, et al. Cystic retroperitoneal lymphangioma in infants and children. Rad 1978;127:203-208. http://dx.doi.org/10.1148/127.1.203

6. Ashraf K, Raza SS, Ashraf $O$, Memon W, Memon A, Zubari TA. Renal lymphangiectasia. British Journal of Radiology 2007;80:e117-118. http://dx.doi. org/10.1259/bjr/16931054

7. Varela JR, Bargiela A, Requejo I, Fernandez R, Darriba M, Pombo F. Bilateral renal lymphangiomatosis: US and CT findings. Eur Radiol 1998;8:230-231. http:// dx.doi.org/10.1007/s003300050368

8. Kim JK, Ahn HJ, Kim KR, Cho KS. Renal lymphangioma manifested as a solid mass on ultrasonography and computed tomography. J Ultrasound Med 2002;21:203-206.

9. Ramseyer LT. Renal lymphangiectasia. Rad 2001;219:442-444. http://dx.doi. org/10.1148/radiology.219.2.r01ma17442 